\title{
Rabi oscillations, Ramsey fringes and spin echoes in an electrical circuit
}

\author{
D. Vion, A. Aassime, A. Cottet, P. Joyez, H. Pothier, \\ C. Urbina, D. Esteve and M.H. Devorett \\ Quantronics Group, Service de Physique de l'Etat Condensé, \\ Département des Sciences de la Matière, CEA-Saclay \\ 91191 Gif-sur-Yvette, France
}

\begin{abstract}
We present a superconducting tunnel junction circuit which behaves as a controllable atom, and whose ground and first excited state form an effective spin 1/2. By applying microwave pulses, we have performed on this circuit experiments demonstrating the controlled manipulation of the spin : Rabi precession, Ramsey interferences, and spin echoes.
\end{abstract}

The state variables of an electrical circuit, like voltages and currents can be made to behave quantum mechanically by minimizing the coupling to external degrees of freedom through a proper design. Circuits based on superconducting tunnel junctions have displayed signatures of macroscopic quantum behavior [1, 2, 3, 4, 5, 6, 7, but the level of coherence of the quantum states remained until now much smaller than for isolated atoms or ions. In the "quantronium" circuit presented here, a coherence quality factor of more than $10^{4}$ has been obtained, thus allowing the coherent manipulation of the state of the system like in atomic physics and NMR experiments [8].

The quantronium consists of a superconducting loop interrupted by two adjacent small Josephson tunnel junctions with capacitance $C_{j} / 2$ and Josephson energy $E_{J} / 2$ each, which define a low capacitance superconducting electrode called the "island", and by a large Josephson junction with large Josephson energy $\left(E_{J 0} \approx 20 E_{J}\right)$ (see Fig. 1). The island is biased by a voltage source $U$ through a gate capacitance $C_{g}$. In addition to $E_{J}$, the quantronium has a second energy scale which is the Cooper pair Coulomb energy $E_{C P}=(2 e)^{2} / 2\left(C_{g}+C_{j}\right)$. The temperature $T$ and the superconducting gap $\Delta$ satisfy $k_{B} T \ll$ $\Delta / \ln \mathcal{N}$ and $E_{C P}<\Delta-k_{B} T \ln \mathcal{N}$, where $\mathcal{N}$ is the total number of paired electrons in the island. The number of excess electrons is then even [9, 10], and the system has discrete quantum states which are in general quantum superpositions of several charge states with different number $\hat{N}$ of excess Cooper pairs in the island. Neglecting the loop inductance and the charging energy of the large junction, the Hamiltonian of the circuit is

$$
\hat{H}=E_{C P}\left(\hat{N}-N_{g}\right)^{2}-E_{J} \cos \left(\frac{\hat{\gamma}+\phi}{2}\right) \cos \hat{\theta}-E_{J 0} \cos \hat{\gamma}-I_{b} \varphi_{0} \hat{\gamma},
$$

where $N_{g}=C_{g} U / 2 e$ is the dimensionless gate charge, $\phi=\Phi / \varphi_{0}$ is a phase bias, with $\Phi$ the external flux imposed through the loop and $\varphi_{0}=\hbar / 2 e, \hat{\gamma}$ is the phase across the large junction, and $\hat{\theta}$ is the phase operator conjugate to the Cooper pair number $\hat{N}$. The bias current $I_{b}$ is zero except during readout of the state [11].

In our experiment $E_{J} \simeq E_{C P}$ and neither $\hat{N}$ nor $\hat{\theta}$ is a good quantum number. In contrast, the large junction is shunted by a large capacitor $C$ so that $\hat{\gamma}$ is almost a classical variable. In this regime, the energy spectrum is sufficiently anharmonic for the two lowest energy states $|0\rangle$ and $|1\rangle$ to form a two-level system. This system corresponds to an effective spin one-half $\vec{s}$ with eigenstates $|0\rangle \equiv\left|s_{z}=+1 / 2\right\rangle$ and $|1\rangle \equiv\left|s_{z}=-1 / 2\right\rangle$. At $N_{g}=1 / 2, I_{b}=0$ and $\phi=0$, its "Zeeman energy" $h \nu_{01}$, of the order of $E_{J}$, is stationary with respect to $N_{g}, I_{b}$ and $\phi$ [8], making the system immune to first order fluctuations of the control parameters. Manipulation of the quantum state is thus performed at this optimal point

*Present address: Applied Physics Department, Yale University, New Haven, CT 06520, USA 


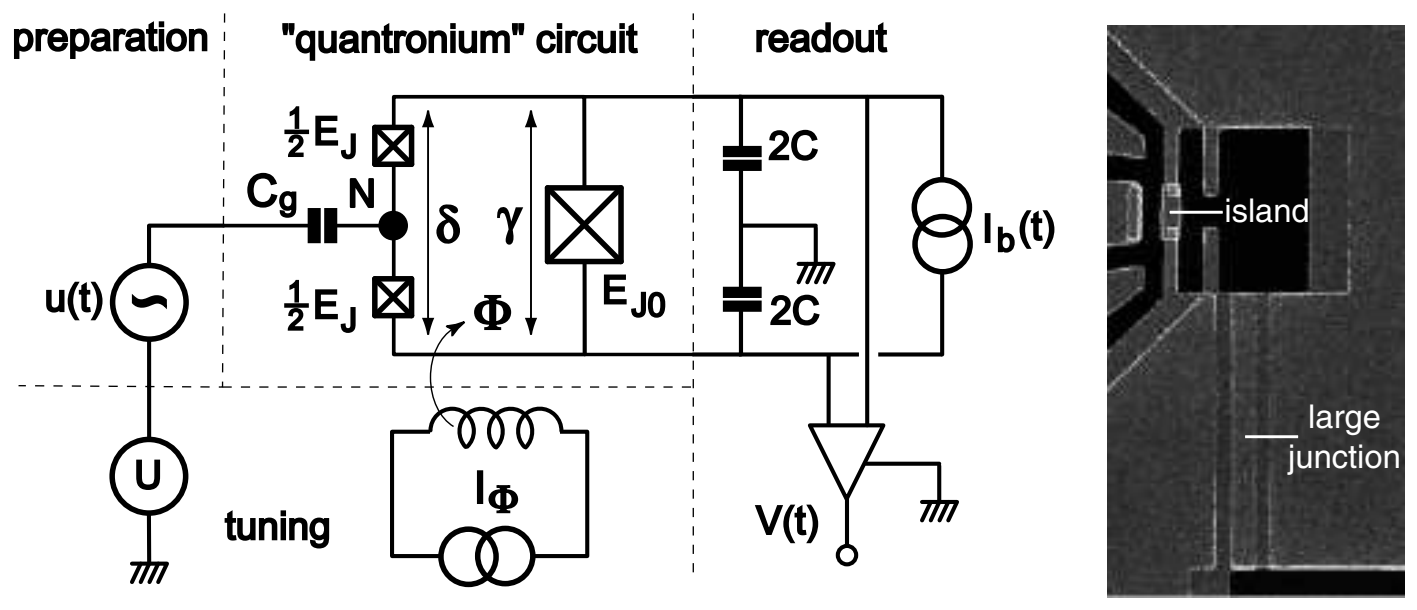

Figure 1: Left : Idealized circuit diagram of the "quantronium", a quantum coherent circuit with its tuning, preparation and readout blocks. The circuit consists of an island (black node) delimited by two small Josephson junctions (crossed boxes) in a superconducting loop. The loop also includes a third, much larger Josephson junction shunted by a capacitance $C$. The Josephson energies of the island and of the large junction are $E_{J}$ and $E_{J 0}$. The Cooper pair number in the island $N$ and the phases $\delta$ and $\gamma$ are the degrees of freedom of the circuit. A dc voltage $U$ applied to the gate capacitance $C_{g}$ and a dc current $I_{\phi}$ applied to a coil producing a flux $\Phi$ in the circuit loop tune the quantum energy levels. Microwave pulses $u(t)$ applied to the gate prepare arbitrary quantum states of the circuit. The states are readout by applying a current pulse $I_{b}(t)$ to the large junction and by monitoring the voltage $V(t)$ across it. Right: Scanning electron micrograph of a sample.

by applying microwave pulses $u(t)$ with frequency $\nu \simeq \nu_{01}$ to the gate, and any superposition $|\Psi\rangle=$ $\alpha|0\rangle+\beta|1\rangle$ can be prepared, starting from $|0\rangle$. In a frame rotating around the quantization axis $z$ at frequency $\nu$, the microwave voltage acts on $\vec{s}$ as an effective dc magnetic field in the $x z$ plane, with $x$ and $z$ components proportional to the microwave amplitude and to the detuning $\Delta \nu=\nu-\nu_{01}$, respectively.

For readout, we have implemented a strategy reminiscent of the Stern and Gerlach experiment [12], in which the information about the spin of silver atoms is transferred onto their transverse position. In our experiment, the information on $\vec{s}$ is transferred onto the phase $\hat{\gamma}$, and the two states are discriminated through the supercurrent in the loop $\langle\hat{I}\rangle=\langle\partial \hat{H} / \partial \hat{\delta}\rangle / \varphi_{0}$, where $\hat{\delta}=\hat{\gamma}+\phi$ is the phase difference across the series combination of the two small junctions [13, 14, 15]. For this purpose, a trapezoidal readout pulse $I_{b}(t)$ with a peak value slightly below the critical current $I_{0}=E_{J 0} / \varphi_{0}$ is applied to the circuit (see Fig. 2).

When starting from $\langle\hat{\delta}\rangle \approx 0$, the phases $\langle\hat{\gamma}\rangle$ and $\langle\hat{\delta}\rangle$ grow during the current pulse, and consequently an $\vec{s}$-dependent supercurrent develops in the loop. The loop current is the analog of the transverse acceleration experienced by a silver atom in the magnetic field gradient. This current adds to the biascurrent in the large junction, and by precisely adjusting the amplitude and duration of the $I_{b}(t)$ pulse, the large junction switches during the pulse to a finite voltage state with a large probability $p_{1}$ for state $|1\rangle$ and with a small probability $p_{0}$ for state $|0\rangle[13$. A switching event corresponds to the impact of a silver atom on the top spot of the screen. The absence of switching corresponds to the impact of a silver atom on the bottom spot of the screen. For the parameters of the experiment, the efficiency of this projective measurement should be $\eta=p_{1}-p_{0}=0.95$ for optimum readout conditions. Large ratios $E_{J 0} / E_{J}$ and $C / C_{j}$ provide further protection from the environment.

An actual "quantronium" sample is shown on the right side of Fig. 1. It was fabricated with standard technique of aluminum evaporation through a shadow-mask obtained by e-beam lithography. With an external microwave capacitor $C=1 \mathrm{pF}$, the plasma frequency of the large junction with $I_{0}=0.77 \mu \mathrm{A}$ is $\omega_{p} / 2 \pi \simeq 8 \mathrm{GHz}$. The sample and last filtering stage were anchored to the mixing chamber of a dilution refrigerator with $15 \mathrm{mK}$ base temperature. The switching of the large junction to the voltage state is detected by measuring the voltage across it with a room temperature preamplifier followed by a discriminator with a threshold voltage $V_{t h}$ well above the noise level (Fig. 2). By repeating the 


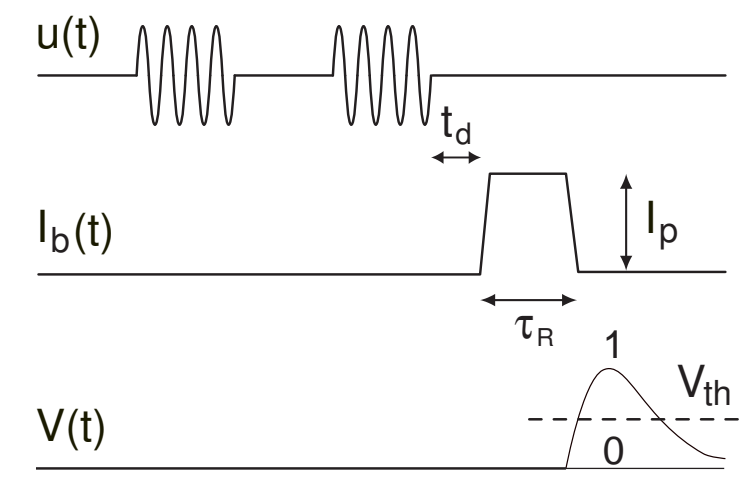

Figure 2: Signals involved in quantum state manipulations and measurement of the "quantronium". Top: microwave voltage pulses are applied to the gate for state manipulation. Middle: a readout current pulse $I_{b}(t)$ with amplitude $I_{p}$ is applied to the large junction $t_{d}$ after the last microwave pulse. Bottom: voltage $V(t)$ across the junction. The occurence of a pulse depends on the occupation probabilities of the energy eigenstates. A discriminator with threshold $V_{t h}$ converts $V(t)$ into a boolean $0 / 1$ output for statistical analysis.

experiment (typically a few $10^{4}$ times), we can determine the switching probability, and hence, the occupation probabilities $|\alpha|^{2}$ and $|\beta|^{2}$.

The readout part of the circuit was tested by measuring the switching probability $p$ as a function of the pulse height $I_{p}$, for a current pulse duration of $\tau_{r}=100 \mathrm{~ns}$, at thermal equilibrium . The discrimination between the currents corresponding to the $|0\rangle$ and $|1\rangle$ states was found to have an efficiency of $\eta=0.6$, which is lower than the expected $\eta=0.95$. Measurements of the switching probability as a function of temperature and repetition rate indicate that the discrepancy between the theoretical and experimental readout efficiency could be due to an incomplete thermalization of our last filtering stage in the bias current line.

Spectroscopic measurements of $\nu_{01}$ were performed by applying to the gate a weak continuous microwave irradiation suppressed just before the readout current pulse. The variations of the switching probability as a function of the irradiation frequency display a resonance whose center frequency evolves as a function of the dc gate voltage and flux as the Hamiltonian (1) predicts, reaching $\nu_{01} \simeq 16.5 \mathrm{GHz}$ at the optimal working point (see Fig. 3). The small discrepancy between theoretical and experimental values of the transition frequency at nonzero magnetic flux is attributed to flux penetration in the small junctions not taken into account in the model. We have used these spectroscopic data to precisely determine the relevant circuit parameters and found $i_{0}=18.1 \mathrm{nA}$ and $E_{J} / E_{C P}=1.27$. The linewidth $\Delta \nu_{01}$ is given in the bottom panels in Fig. 3 as a function of $\phi$ and $N_{g}$. At the optimal working point, the linewidth was found to be minimal with a $0.8 \mathrm{MHz}$ full width at half-maximum, corresponding to a quality factor $Q=2 \times 10^{4}$. The lineshape was found to be irreproducible, probably because of slight shifts of the resonance frequency during the measurement, related to low frequency charge or noise on the phase $\delta$. If one considers the narrowest lines recorded, the linewidth varies linearly when departing from the optimal point $\left(N_{g}=1 / 2, \phi=0, I_{b}=0\right)$, the proportionality coefficients being $\partial \Delta \nu_{01} / \partial N_{g} \simeq 250 \mathrm{MHz}$ and $\partial \Delta \nu_{01} / \partial(\phi / 2 \pi) \simeq 430 \mathrm{MHz}$. These values can be translated into RMS deviations $\Delta N_{g}=0.004$ and $\Delta(\delta / 2 \pi)=0.002$ during the time needed to record the resonance. The residual linewidth at the optimal working point can be explained by the second order contribution of these noises. The amplitude of the charge noise is in agreement with measurements of $1 / f$ charge noise [16], and its effect could be minimized by increasing the $E_{J} / E_{C}$ ratio. By contrast, the amplitude of the phase noise corresponds to a large flux noise [17, but it could be also attributed to bias current noise.

When varying the delay between the end of a resonant irradiation and the measurement pulse at the optimal working point, the switching probability decays with a time constant $T_{1}=1.8 \mu$ s (see Fig. 4). Supposing that the energy relaxation of the system is only due to the bias circuitry, a calculation along the lines of Ref. [19] predicts that $T_{1} \sim 10 \mu$ s for a crude discrete element model. This result shows that no detrimental sources of dissipation have been seriously overlooked in our circuit design.

We have then performed controlled rotations of $\vec{s}$ with large amplitude microwave pulses. Prior to readout, a single pulse at the transition frequency with variable amplitude $U_{\mu w}$ and duration $\tau$ was 


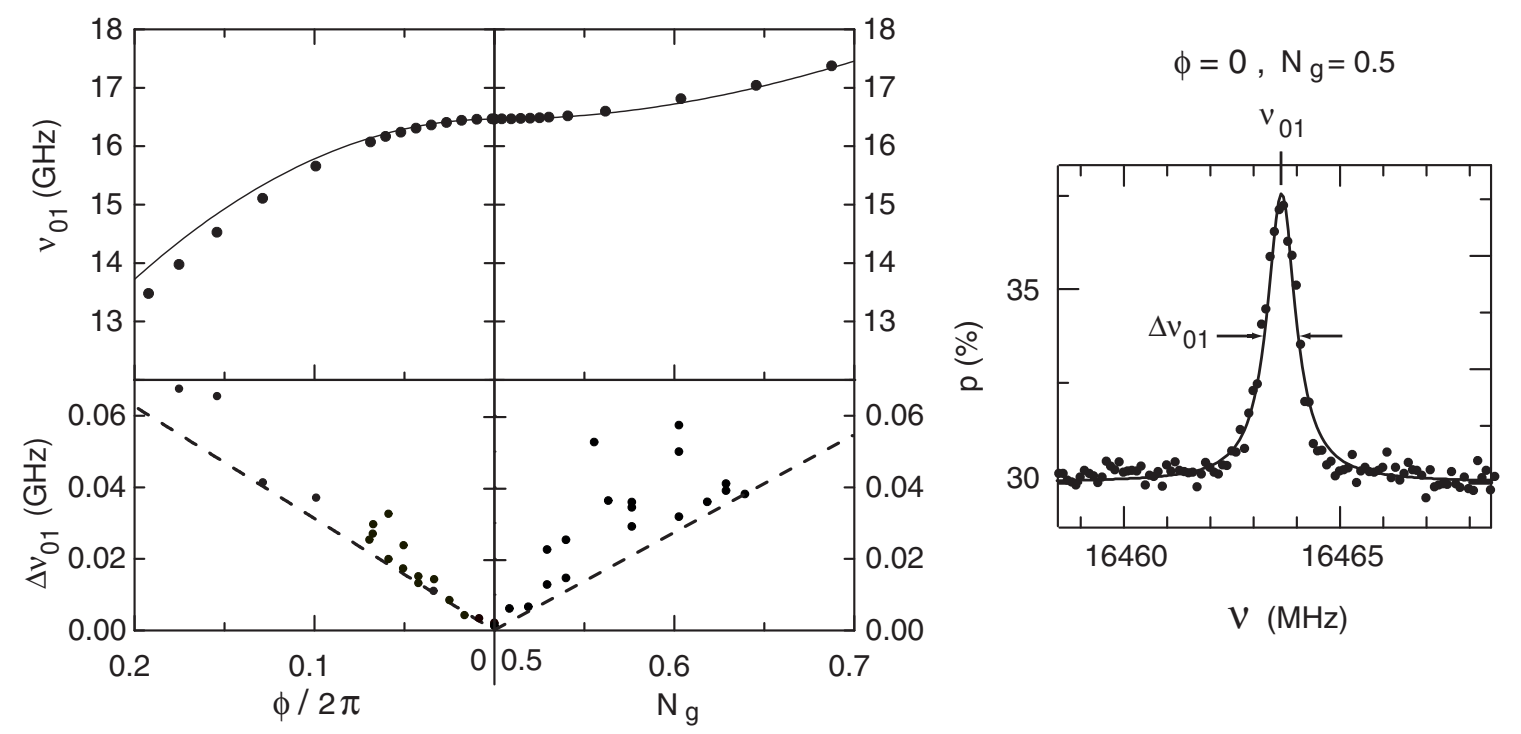

Figure 3: Left: Measured center frequency $\nu_{01}$ (top panels, symbols) and full width at half maximum $\Delta \nu_{01}$ (bottom panels, symbols) of the resonance as a function of reduced gate charge $N_{g}$ for reduced flux $\phi=0$ (right panels), and as a function of $\phi$ for $N_{g}=0.5$ (left panels), at $15 \mathrm{mK}$. Spectroscopy is performed by measuring the switching probability $p$ when a continuous microwave irradiation of variable frequency is applied to the gate before readout $\left(t_{d}<100 \mathrm{~ns}\right)$. Continuous lines in top panels: theoretical best fit (see text). Dotted lines in bottom panels correspond to $\partial \Delta \nu_{01} / \partial N_{g} \simeq 250 \mathrm{MHz}$ and $\partial \Delta \nu_{01} / \partial(\phi / 2 \pi) \simeq 430 \mathrm{MHz}$. They give a lower bound to the measured $\Delta \nu_{01}$. Right: Lineshape measured at the optimal working point $\phi=0$ and $N_{g}=0.5$ (dots). Lorentzian fit with a FWHM $\Delta \nu_{01}=0.8 \mathrm{MHz}$ and a center frequency $\nu_{01}=16463.5 \mathrm{MHz}$ (solid line).

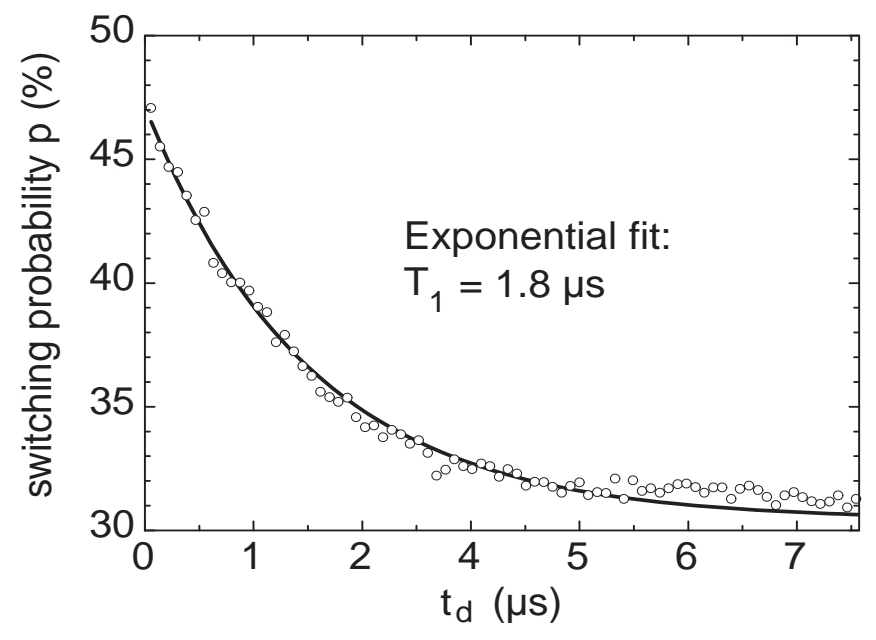

Figure 4: Decay of the switching probability as a function of the delay time $t_{d}$ after a continuous excitation at the center frequency of the resonance line. The solid line is an exponential fit (vertically offset by the signal measured without microwave applied to the gate), from which the relaxation time $T_{1}=1.8 \mu \mathrm{s}$ is obtained. 


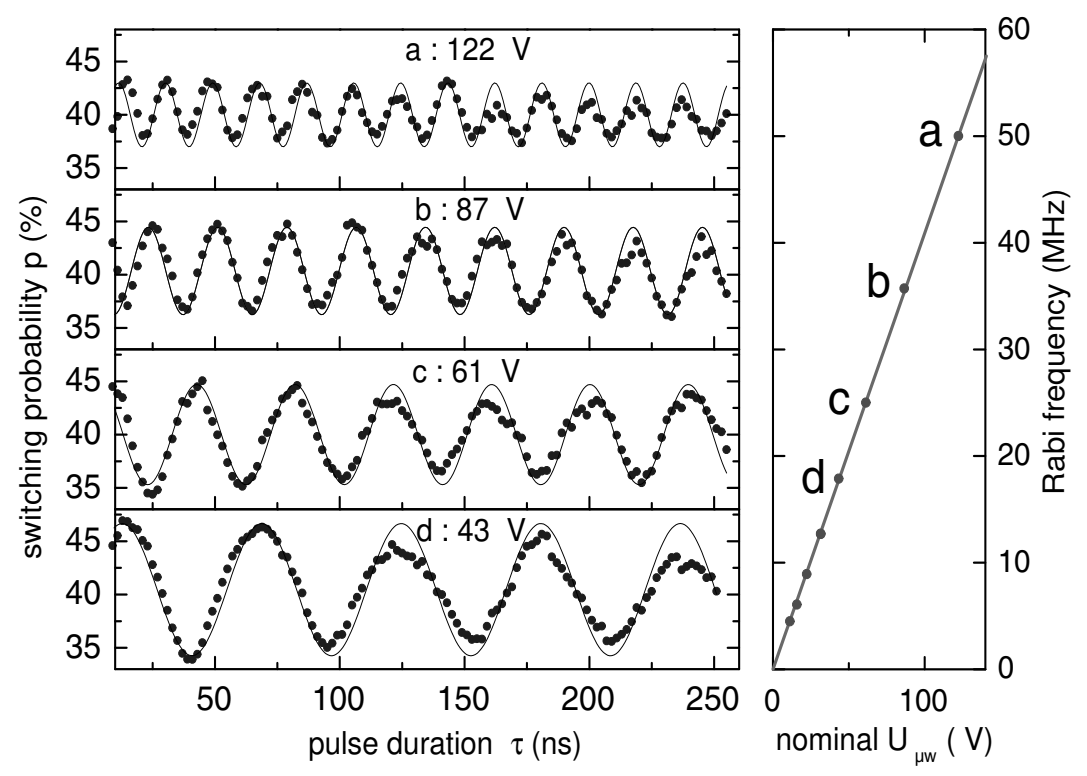

Figure 5: Left: Rabi oscillations of the switching probability $p\left(5 \times 10^{4}\right.$ events $)$ measured just after a resonant microwave pulse of duration $\tau$. Data taken at $15 \mathrm{mK}$ for nominal pulse amplitudes $U_{\mu w}=122$, 87,61 , and $43 \mu \mathrm{V}$ (dots, from top to bottom). The Rabi frequency is extracted from sinusoidal fits (continuous lines). Right: the Rabi frequency (dots) varies linearly with $U_{\mu w}$, as expected. The four points labelled $a$ to $d$ correspond to the curves on the left.

applied. The resulting change in switching probability is an oscillatory function of the product $U_{\mu w} \tau$ (see Fig. 5), in agreement with the theory of Rabi oscillations [18]. It provides direct evidence that the resonance indeed corresponds to an effective spin rather than to a spurious harmonic oscillator resonance in the circuit. The proportionality ratio between the Rabi period and $U_{\mu w} \tau$ was used to calibrate microwave pulses for the application of controlled rotations of $\vec{s}$.

The measurement of the coherence time of $\vec{s}$ during free evolution was obtained by performing a Ramsey-fringes-like experiment [20]. One applies on the gate two phase coherent microwave pulses corresponding each to a $\pi / 2$ rotation around $x$ 21] and separated by a delay $\Delta t$ during which the spin precesses freely around $z$. For a given detuning $\Delta \nu$ of the microwave frequency, the switching probability displays decaying oscillations of frequency $\Delta \nu$ (see Fig. 6), which correspond to the "beating" of the spin precession with the external microwave field. The envelope of the oscillations yields the coherence time $T_{\varphi} \simeq 0.5 \mu \mathrm{s}$. Given the transition period $1 / \nu_{01} \simeq 60 \mathrm{ps}$, this means that $\vec{s}$ can perform on average 8000 coherent free precession turns.

When the circuit is biased away from the optimal point, the coherence time $T_{\varphi}$ of the oscillation is strongly reduced, as shown in the top panel of Fig. 7 for $N_{g}=1 / 2+0.02, \phi=0$. In order to determine the contribution to dephasing of low frequency charge noise, we have performed spin echo experiments: an intermediate $\pi$ pulse is inserted between the two $\pi / 2$ pulses of the Ramsey sequence (see Fig. 7 , right side). The effect of the $\pi$ pulse is to make the phases accumulated during the two free evolution time intervals $t_{1}$ and $t_{2}$ to subtract one from the other (see Fig. 7). By symmetry, when $t_{1}=t_{2}$, the total phase accumulated is independent of $\Delta \nu$ if $\Delta \nu$ is constant over the complete sequence. Compared to the Ramsey fringes experiment, where coherence during $\Delta t$ is revealed by the periodic evolution of $\langle\cos [2 \pi \Delta \nu \Delta t]\rangle$, the echo signal varies as $\left(1-\left\langle\cos \left[2 \pi \Delta \nu\left(t_{2}-t_{1}\right)\right]\right\rangle\right) / 2$ and is therefore less sensitive to fluctuations of $\Delta \nu$ from sequence to sequence when $t_{1} \sim t_{2}$. In the experiment, we have recorded the switching probability at fixed values of $\Delta t$, as a function of the delay $t_{1}$ (left panels of Fig. 7). Up to $\Delta t \simeq 1 \mu \mathrm{s}$, fringes emerge around $t_{1}=t_{2}=(\Delta t-\tau) / 2$ (here, $\tau \sim 15 \mathrm{~ns}$ ), indicating that during pulse sequences of this duration, coherence was at least partly conserved. As expected, the period of the oscillations is twice as short in the echo experiment than in the Ramsey experiment. The observation of spin echoes at time scales much larger than the decay time of the Ramsey fringes indicates that in this situation decoherence was essentially due to charge fluctuations at frequencies lower than $1 / \Delta t \approx 1 \mathrm{MHz}$. No echo was seen in 


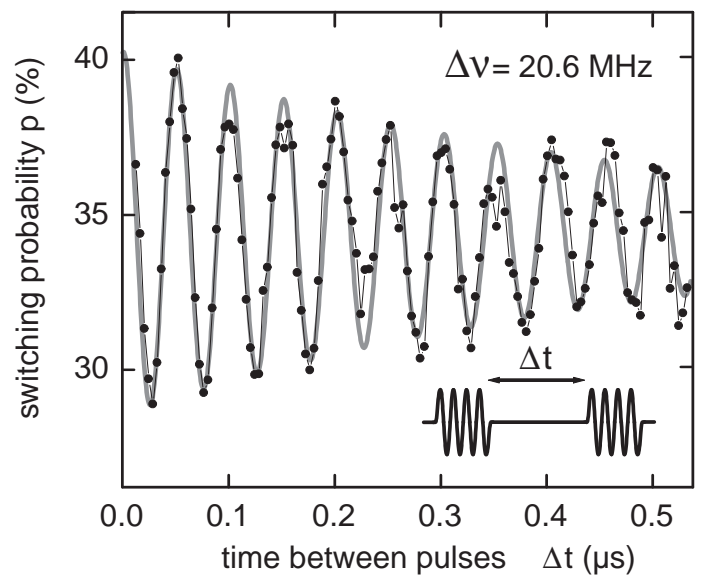

Figure 6: Ramsey fringes of the switching probability $p$ after two $\pi / 2$ microwave pulses separated by $\Delta t$. Dots: data at $15 \mathrm{mK}$. The total acquisition time was $5 \mathrm{mn}$. Continuous grey line: fit by exponentially damped sinusoid with frequency $20.6 \mathrm{MHz}$, equal to the detuning frequency $\Delta \nu$, and decay time constant $T_{\varphi}=0.5 \mu \mathrm{s}$.

experiments performed at $\phi \neq 0$, suggesting that the relevant phase noise was at higher frequencies.

In all our time domain experiments, the oscillation period of the switching probability closely agrees with theory, meaning a precise control of the preparation of $\vec{s}$ and of its evolution. However, the amplitude of the oscillations is smaller than expected by a factor of three to four. This loss of contrast is likely to be due to a relaxation of the level population during the measurement itself. In principle the current pulse, whose rise time is $50 \mathrm{~ns}$, is sufficiently adiabatic not to induce transitions directly between the two levels. Nevertheless, it is possible that the readout or even the preparation pulses excite resonances in the bias circuitry which in turn could induce transitions in our two-level manifold. Experiments using better shaped readout pulses and a bias circuitry with better controlled high-frequency impedance are needed to clarify this point.

In conclusion we have designed and operated a superconducting tunnel junction circuit which behaves as a tunable two-level atom that can be decoupled from its environment. When the readout is off, the coherence of this "quantronium" atom is of sufficient quality $\left(Q_{\varphi}=2.5 \times 10^{4}\right)$ that an arbitrary quantum evolution can be programmed with a series of microwaves pulses. Coupling several of these circuits can be achieved using on-chip capacitors. The ability to tune and address them individually would allow to produce entangled states and probe their quantum correlations. These fundamental physics experiments could lead to the realization of quantum logic gates, an important step towards the practical implementation of solid-state quantum processors [22.

Acknowledgements: The indispensable technical work of Pief Orfila is gratefully acknowledged. This work has greatly benefited from direct inputs from J. M. Martinis and Y. Nakamura. The authors acknowledge discussions with P. Delsing, G. Falci, D. Haviland, H. Mooij, R. Schoelkopf, G. Schön and G. Wendin. This work is partly supported by the European Union through contract IST-10673 SQUBIT and by the Conseil Général de l'Essonne (EQUM project).

\section{References}

[1] M. H. Devoret et al, in Quantum Tunneling in Condensed Media edited by Y. Kagan and A.J. Leggett (Elsevier Science Publishers, 1992).

[2] V. Bouchiat, D. Vion, P. Joyez, D. Esteve, and M. H. Devoret, Phys. Scr. T76, 165 (1998). 

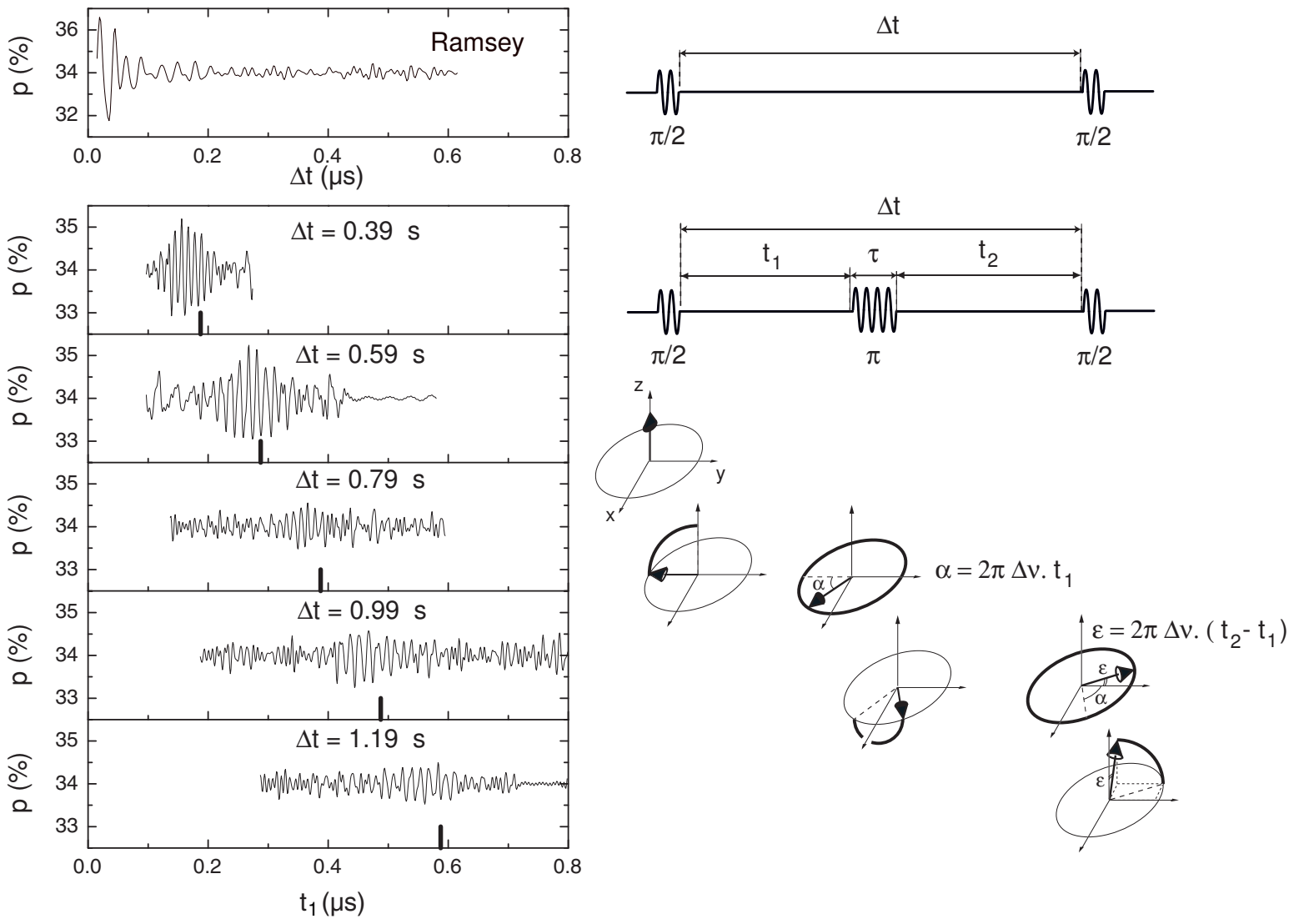

Figure 7: Top panel: Ramsey fringes measured at $N_{g}=0.52, \phi=0$ and $\Delta \nu=41 \mathrm{MHz}$. The decay time constant of the fringes is here $T_{\varphi} \sim 30 \mathrm{~ns}$. Lower panels : echo signals obtained with the pulse sequence schematically described on the right side, for various sequence durations $\Delta t$. A first $\pi / 2$ pulse brings the spin $\vec{s}$ on the $-y$ axis. Follows a free precession by an angle $\alpha=2 \pi \Delta \nu t_{1}$ during a time $t_{1}$. A subsequent $\pi$ pulse brings the spin in the symmetric position with respect to $x$ axis. Follows a second free precession during time $t_{2}$, which brings the spin at an angle $\varepsilon=2 \pi \Delta \nu\left(t_{2}-t_{1}\right)$ with the $y$ axis. The last $\pi / 2$ pulse results in a final $z$ component of the spin equal to $\cos \varepsilon$. The average switching probability $p=(1-\langle\cos \varepsilon\rangle) / 2$, obtained by repeating the sequence, is an oscillating function of $t_{2}-t_{1}$. The amplitude of the oscillations is damped away from $t_{1}=t_{2}$ (thick tick in each panel) due to fluctuations of $\Delta \nu$. 
[3] Y. Nakamura, C. D. Chen, and J. S. Tsai, Phys. Rev. Lett. 79, 2328 (1997); Y. Nakamura, Yu. A. Pashkin, J. S. Tsai, Nature 398, 786 (1999); Y. Nakamura, Yu. A. Pashkin, T. Yamamoto, J. S. Tsai, Phys. Rev. Lett. 88, 047901 (2002).

[4] C. H. van der Wal et al, Science 290, 773 (2000).

[5] Siyuan Han, R. Rouse, J. E. Lukens, Phys. Rev. Lett. 84, 1300 (2000).

[6] Siyuan Han, Yang Yu, Xi Chu, Shih-I Chu, Zhen Wang, Science 293, 1457 (2001).

[7] J. M. Martinis, S. Nan, J. Aumentado, C. Urbina (unpublished) have recently obtained $Q_{\varphi}$ 's reaching 1000 for a current biased Josephson junction.

[8] D. Vion, A. Aassime, A. Cottet, P. Joyez, H. Pothier, C. Urbina, D. Esteve, and M.H. Devoret, Science 296, 886 (2002).

[9] M. T. Tuominen, J. M. Hergenrother, T. S. Tighe, M. Tinkham, Phys. Rev. Lett. 69, 1997 (1992).

[10] P. Lafarge, P. Joyez, D. Esteve, C. Urbina, M.H. Devoret, Nature 365, 422 (1993).

[11] V. Bouchiat, D. Vion, P. Joyez, D. Esteve, M. H. Devoret, Phys. Scr. T76, 165 (1998).

[12] O. Stern and W. Gerlach, Z. Phys. 8, 110 (1922).

[13] A. Cottet, D. Vion, P. Joyez, A. Aassime, D. Esteve, M. H. Devoret, Physica C 367, 197 (2002).

[14] Another two-port design has been proposed by A. B. Zorin, Physica C 368, 284 (2002).

[15] A different readout scheme using a large Josephson junction is discussed by F. W. J. Hekking, O. Buisson, F. Balestro, and M. G. Vergniory, in Electronic Correlations: from Meso- to Nanophysics, T. Martin, G. Montambaux and J. Trân Thanh Vân, eds. (EDP Sciences, 2001), p. 515.

[16] H. Wolf et al, IEEE Trans. on Instrum. and Measurement 46, 303 (1997).

[17] F. C. Wellstood, C. Urbina, J. Clarke, Appl. Phys. Lett. 50, 772 (1987).

[18] I. I. Rabi, Phys. Rev. 51, 652 (1937).

[19] A. Cottet, A. H. Steinbach, P. Joyez, D. Vion, H. Pothier, D. Esteve, and M. E. Huber, in Macroscopic quantum coherence and quantum computing, edited by D. V. Averin, B. Ruggiero, and P. Silvestrini (Kluwer Academic, Plenum publishers, New-York, 2001), p. 111.

[20] N. F. Ramsey, Phys. Rev. 78, 695 (1950).

[21] In practice, the rotation axis does not need to be $x$, but the rotation angle of the two pulses is always adjusted so as to bring a spin initially along $z$ into a plane perpendicular to $z$.

[22] M. A. Nielsen, I. L. Chuang, Quantum Computation and Quantum Information (Cambridge University Press, Cambridge, 2000). 